\title{
Framing a Transdisciplinary Research Agenda in Health Education to Address Health Disparities and Social Inequities: A Road Map for SOPHE Action
}

\author{
Stephen F. Gambescia, PhD, MEd, MBA, CHES \\ Lynn D. Woodhouse, MEd, EdD, MPH, CHES \\ M. Elaine Auld, MPH, CHES \\ B. Lee Green, PhD \\ Sandra Crouse Quinn, $\mathrm{PhD}$ \\ Collins O. Airhihenbuwa, PhD, MPH
}

\begin{abstract}
SOPHE leaders continue to challenge us to be true to the call for an "open society." SOPHE has supported the Healthy People 2010 goal of eliminating health disparities through its Strategic Plan. SOPHE held an Inaugural Health Education Research Disparities Summit, Health Disparities and Social Inequities: Framing a Transdisciplinary Research Agenda in Health Education, August 8 and 9, 2005. This article explains the process used at the Summit where more than 80 researchers, academicians, practitioners, and students from across the country convened to ask fundamental questions about health disparity associated with race and ethnicity and how a health education research agenda could help in eliminating these disparities. From this Summit, about a dozen questions and/or recommendations have been developed to frame our future discussions about health disparities. Through its Research Agenda Committee, SOPHE has developed a process of translation and dissemination, including community participation, review, dialogue, and action.
\end{abstract}

Keywords: health disparities; health education research; SOPHE's health education research agenda; transdisciplinary research

Of all the forms of inequality, injustice in health is the most shocking and inhumane.

—Reverend Martin Luther King (cited in Freeman, 1989)

Health for all, social justice, and equality are embodied in the philosophy, principles, and core values of the health education profession. Few health educators survive the thick

Stephen F. Gambescia, Drexel University, Philadelphia, Pennsylvania. Lynn D. Woodhouse, East Stroudsburg University, Pennsylvania. M. Elaine Auld, Society for Public Health Education, Washington, D.C. B. Lee Green, Center for the Study of Health Disparities, Texas A\&M University, College Station. Sandra Crouse Quinn, Graduate School of Public Health, University of Pittsburgh, Pennsylvania. Collins O. Airhihenbuwa, Pennsylvania State University, University Park.

Address reprint requests to Stephen F. Gambescia, $\mathrm{PhD}$, MEd, MBA, CHES, President, Society for Public Health Education, Drexel University, Philadelphia, PA 19104; phone: 610-789-6795; e-mail runswim@ comcast.net. 
and thorny vineyard of public health without a passionate commitment to eliminating health disparities associated with race, ethnicity, language, gender, sexual orientation, or geography.

"Promoting the health of ALL people through education" has been an organizational tagline of the Society for Public Health Education (SOPHE) since its inception. Yet it was not until 1967 that SOPHE President Dorothy Nyswander (1982) openly challenged SOPHE to achieve the vision of an open society. More recently, this call was resurrected by Dr. Kathleen Roe in 1998, when as SOPHE president, she appointed the SOPHE Open Society Commission to pursue the quest of an environment in which

Justice is the same for every man; where dissent is taken seriously as an index of something wrong or something needed; where diversity is respected; where pressure groups cannot stifle and control the will of the majority or castigate the individual; where education brings upward mobility to all; where the best of health care is available to all; where poverty is a community disgrace not an individual's weakness; where greed for possessions and success is replaced by inner fires for excellence and honor; where desires for power over men become satisfaction with the use of power for men.

Since then, SOPHE has supported the Healthy People 2010 goal of eliminating health disparities through its Strategic Plan, which has served as a road map for organizational efforts such as:

- advocating and testifying for appropriations for programs such as Centers for Disease Control and Prevention's (CDC) Racial and Ethnic Approaches to Community Health and the NIH National Center for Minority Health and Health Disparities;

- addressing research and best practices related to eliminating health disparities as a major theme and/or subtheme in every SOPHE Midyear or Annual Meeting since 1999;

- adopting a series of resolutions on elimination of health disparities due to race, ethnicity, gender, geography, disability status, and sexual orientation; and

- braiding public and private sector funds to sponsor five special journal issues and supplements on eliminating health disparities in various populations since 2002, including one of the first issues on eliminating racial and ethnic health disparities in Health Promotion Practice (Roe $\&$ Thomas, 2002). In that same issue, 92 minority health resources from the Office of Minority Health were featured, followed by another issue that published brief reviews of the collective Jossey-Bass works on understanding race, ethnicity, and health (Gambescia, 2002, 2005).

Given SOPHE's commitment to an open society and to advancing the theoretical basis of the field, a natural next step was to develop a health education research agenda focused on eliminating health disparities. SOPHE's existing research agenda, published in 1995, devoted only one chapter to health issues in "special populations" (Clark \& McLeroy, 1995) and was clearly dated in terms of research methods and focus. Thus, SOPHE's 2005 president, Dr. Collins Airhihenbuwa, successfully marshaled the funding and the will in record time to sponsor SOPHE's Inaugural Health Education Research Disparities Summit, Health Disparities and Social Inequities: Framing a Transdisciplinary Research Agenda in Health Education held August 8 and 9, 2005, in Alexandria, Virginia. Given the complexity of issues related to health disparities, this inaugural Summit focused on health disparity associated with race and ethnicity. Although SOPHE is proud for taking the lead in this important public health area, it also recognizes the tremendous responsibility; for "inaugural" not only means the first but also means the first in a series of activities. 
An expert transdisciplinary committee planned the objectives, outcomes, and focus of the 2-day meeting, which convened more than 80 researchers, academicians, practitioners, and students from across the country. As required reading, participants were given the assignment to review several important documents related to health disparities. Of note are the Institute of Medicine's (2002) unequal treatment among race and ethnicities report; the Agency for Healthcare Research and Quality's (2004) report on health care disparities in the United States; and the Louis Sullivan Commission's report on minorities in the health professions (Sullivan Commission, 2004). Participants soon became acutely aware that much time and energy has already been devoted to identifying the nature and extent of health disparities among racial and ethnic groups in the United States; however, still there remains a paucity of solutions and action.

The Summit was highly participatory, collaborative, and consensual. Four plenary sessions involving distinguished experts addressed key Summit questions:

- If community participation is central to the elimination of health disparities, what social ecological anchors, policies, cultures, and other contexts are essential for health education program success?

- Based on what is known about the problem of health disparities and what seems to work in health education, what are some ways in which to disseminate this information to researchers and engage the community in developing evidenced-based interventions?

- What are the research gaps in what we don't know about addressing health disparities among racial and ethnic groups?

Following the plenary sessions, Summit participants worked in five groups on 10 topical areas to assess what is known in health education about how to eliminate racial and ethnic health disparities, identify gaps, and establish priorities for establishing meaningful health education research in this area. These groups were led by cofacilitators, with a rapporteur and recorder assigned to document contributions from the members. The 10 topics for the work groups included social context and culture, access to services and resources, data and information sharing, policy and workforce, transdisciplinary strategies, dissemination and/or translation to community, dissemination and/or translation to policy makers, health literacy, expanding program evaluation, and exploring new qualitative methods.

After the working groups' sessions of more than 2 hours, the Summit participants reconvened in a general session. The cofacilitators of the groups first gave a briefing about the process, members' exchange, and strategy of their session. This was followed by reports of observations, issues, and recommended research questions from the work group. The issues and/or questions were transcribed into an electronic file, and all Summit participants present were asked to rank the issues and/or questions using an electronic (handheld) Delphi voting process. Participants had an opportunity to see the aggregate distribution of ranking (range), thereby learning the priority issues of the full group. Each group generated between 5 and 10 priority questions and issues that were presented for reconsideration by the entire group. Using the same voting methods, the following research questions emerged as the ones considered the highest priority:

- How do economics and the built environment such as the availability of housing and sidewalks affect health, and how we can encourage the urban design and planning of communities to eliminate health disparities?

- How does power operate in different social contexts to create and maintain disparities? 
- What factors exist in certain populations that protect them from major health issues; for example, what can we learn from African American female teens that experience less drug abuse than other teens? How can health educators and society promote such protective factors?

- How can we culturally tailor interventions to influence access to health services?

- How do we engage and partner with policy makers in diffusing relevant research?

- What information are consumers getting on health, and how does this information differ by race, ethnicity, socioeconomic, and cultural group?

- What is the impact of health literacy on health status, and how can we improve message tailoring to reach different groups?

- Does engagement in community-based participatory research alter engagement in community structures, processes, and other attributes?

- How can we develop more evaluation instruments that assess dynamic, changing, and social conditions such as social event history analysis?

- How can we improve the measurement of intended and unintended effects and outcomes in evaluation studies?

It should be noted that these questions and/or recommendations are meant to frame our future discussions about health disparities. They are not meant to be definitive and final but aim to begin the dialogue with communities and policy makers. As such, the questions and/or recommendations may need further debate and discussion for further clarifications. It will be critically important for all stakeholders to provide input on these questions so that we can make the best effort possible for eliminating health disparities.

Following the Summit, the cofacilitators were asked to determine if the original 10 areas (e.g., health literacy, policy, and workforce) still had utility, given the consensus voting process of identifying research priorities. They were asked to identify trends or clusters of recommendations and began the validation process of identifying the working constructs from which to formulate a research agenda. The research agenda is a process of highlighting research areas or questions that need immediate attention and an agenda for training future public health education researchers to adopt research philosophies and methods necessary for the elimination of health disparities.

A critical next step in this process is translation and dissemination. In addition to several SOPHE Midyear and Annual Meetings, the Summit research agenda is being disseminated through presentations to key stakeholders such as American Public Health Association (APHA), National Association of County and City Health Officials (NACCHO), and others. Given the transdisciplinary nature of the work that needs to be done, the Summit's results are being communicated to a wider audience well beyond SOPHE through meeting presentations. Thanks to the Henry J. Kaiser Foundation, the Summit proceedings also were immediately available via Web casting, and their Web site continues to devote space to this issue (www .kff.org).

This next phase of the project is equally critical to support dissemination and translation of the findings into action at the community level. The proceedings and an analysis of the need for competency development in working with disparate communities emerged from the Summit transcripts, and related documents are being used to support the development of a teaching tool and manual. These materials will be disseminated in the form of a CD-ROM collaboratively developed and produced from the generous support of the sponsoring organizations (Society for Public Health Education, 2006b).

The W. K. Kellogg Foundation also has funded a SOPHE proposal to translate the research agenda within communities. Community members must be central participants in health disparities research, working in collaboration with multidisciplinary researchers who represent public health and nonhealth perspectives. Dialogue is now needed at the 
community level among community members, researchers, and practitioners about innovative solutions to addressing racial and ethnic health disparities and how to work collaboratively to develop more context-based qualitative and quantitative measurement tools. Such tools are critical to bridging data gaps related to social context, culture, and power in health disparities investigations. "As researchers, we need to do a better job of communicating the strength that community members bring to disparities research and to forge closer collaboration between research and practice," concluded Summit speaker Nina Wallerstein, DrPH, of the University of New Mexico, who has devoted her career to conducting research with Native American and other disadvantaged populations.

SOPHE has seen the importance and impact of having such a focused Summit on the field and how it can catalyze new collaborations and research approaches. We believe that this research Summit on eliminating health disparities will have an even greater impact on the profession and other disciplines. Health education is a profession that draws from many other disciplines. It is, therefore, in a position to bring together multidisciplinary scholars to frame a strategy for eliminating health disparity that transcends any single discipline. Indeed, our unique approach involves gathering public health scholars, and those representing law, urban planning, history, economics, and so on, to collectively develop a transdisciplinary framework that will help alleviate "silo" investigation of this problem.

The SOPHE Research Agenda Committee (RAC) is dedicating full attention to this issue. Led by B. Lee Green as cochair, the RAC will be examining the issues and/or questions generated by the Summit by convening several "think-tank" sessions during SOPHE Annual and Midyear meetings. The issues and/or questions that the RAC will be examining include how to strengthen the capacity or develop the infrastructure to conduct health disparities research; how to increase the dialogue between the community, practitioners, researchers, and policy makers; how to develop the research and/or practice link related to dissemination and translation; how to develop a set of shared terms related to health disparities; how to develop collaborations and partnerships with other groups and organizations; and how to measure the success of the research agenda as it relates to the elimination of health disparities. These issues can be considered as either process or structural. Two subcommittees are formed to examine issues from a process perspective and from a structural development perspective. The key element of the RAC plans is to engage many people in the discussion of the Summit, RAC questions, and recommendations. This also includes the community and other stakeholders who will be critically important in reviewing and contributing to the work to date. The activities of the RAC will also facilitate the research agenda questions and the agenda to support the development of researchers (beginning and senior) for the future.

It is critical that we not only create our own research agenda but also advocate that the health disparities issue receive attention from the funders of research, private and public. This funding must include projects and initiatives that go beyond the traditional health services issues to the often-neglected preventive and community health education services. SOPHE has received funding from the W. K. Kellogg Foundation to begin the process of working with its local chapters to engage communities and/or policy makers in how best to implement the key Summit recommendations. At the same time, the forthcoming CD-ROM will be distributed to training institutions and key stakeholders to further extend the range of partners in the conversation on the role of social contexts in eliminating health disparities.

SOPHE is well under way in completing another strategic planning cycle. The issue of health disparities among racial and ethnic groups has always received serious attention 
during our strategic planning processes. Eliminating health disparities received a high priority ranking among the core goals set for the 2006-2010 Strategic Plan (Society for Public Health Education, 2006a). Eliminating health disparities must continue to receive significant attention at SOPHE Annual and Midyear meetings. SOPHE encourages each of us to be reflective in our own regard with the issue of health disparities.

The communities across America are on one hand becoming more diverse. However, at a certain level, there is still a challenge to "identify" individuals according to a particular race or ethnicity and by culture or the contexts in which they live. At a time when we aim to be more acutely aware of differences among people, based on the belief that this understanding will support change through a better understanding of any disparities and subsequent public health education interventions, we may actually be looking for differences in an ever-changing kaleidoscope of people and their struggles in different contexts. The issue of race, ethnicity, and culture becomes more complex as we develop into a rich, multiethnic nation. The researchers at the Summit identified these challenges. This discussion will grapple with biological questions, include practical implications, and may even call for philosophical discourse debating realist, nominalist, universalist, or conceptualist perspectives.

As much as we need to identify and segment people according to their biological, social, and cultural characteristics, we must not lose sight of the fact that we are in toto Homo sapiens; each eligible for the same rights, privileges, and care.

\section{References}

Agency for Healthcare Research and Quality. (2004). 2004 national healthcare disparities report. Washington, DC: Author.

Clark, N., \& McLeroy, K. R. (1995). Creating capacity: A research agenda for public health. Health Education Quarterly, 22, 3.

Freeman, H. P. (1989). Cancer in the socioeconomically disadvantaged. CA: A Cancer Journal for Clinicians, 39(5), 266-288.

Gambescia, S. F. (2002). Resources from the Office of Minority Health. Health Promotion Practice, 3, 130-132.

Gambescia, S. F. (2005). Resources for understanding race, ethnicity, and health. Health Promotion Practice, 6, 29-30.

Institute of Medicine. (2002). Unequal treatment: Confronting racial and ethnic disparities in healthcare. New York: National Academies Press.

Nyswander, D. B. (1982). The open society: Its implications for health educators. In The SOPHE heritage collection of health education monographs (Vol. 1, pp. 29-42). Oakland, CA: Third Party Publishing.

Roe, K. M., \& Thomas, S. (2002). Eliminating racial and ethnic health disparities. Health Promotion Practice, 3, 2.

Society for Public Health Education. (2006a). SOPHE's strategic plan 2006-2010. Washington, DC: Author.

Society for Public Health Education. (2006b). Title to be named [CD-ROM]. Washington, DC: Author.

Sullivan Commission. (2004). Missing persons: Minorities in the health professions: A report of the Sullivan Commission on diversity in the healthcare workforce. Washington, DC: Author. 